\title{
Asymmetrical cortical vein sign predicts early neurological deterioration in acute ischemic stroke patients with severe intracranial arterial stenosis or occlusion
}

Wei Li ${ }^{1}$, Wei-Min Xiao ${ }^{1}$, Gen-Pei Luo ${ }^{1}$, Yong-Lin Liu' ${ }^{1}$, Jian-Feng Qu ${ }^{1}$, Xue-Wen Fang ${ }^{2}$, Fang Wang ${ }^{2}$ and Yang-Kun Chen ${ }^{1 *}$

\begin{abstract}
Background: Susceptibility weighted imaging (SWI) provides an approximate assessment of tissue perfusion and shows prominent hypointense cortical veins in the ischemic territory because of the increased concentration of deoxyhemoglobin. We aimed to evaluate whether asymmetrical prominent cortical vein sign (APCVS) on SWI can predict early neurological deterioration (END) in acute ischemic stroke patients with severe intracranial arterial stenosis or occlusion (SIASO).
\end{abstract}

Methods: One hundred and nine acute ischemic stroke patients with SIASO who underwent SWI were retrospectively recruited. END was defined as an increase in the National Institutes of Health Stroke Scale score $\geqq 2$ points despite standard treatment in the first $72 \mathrm{~h}$ after admission. The APCVS was defined as more and/or large vessels with greater signal loss than those in the opposite hemisphere on SWI.

Results: Thirty out of the 109 (27.5\%) patients developed END. Sixty (55.0\%) patients presented with APCVS on SWI. APCVS occurred in 24 (80\%) patients with END, whereas it only occurred in $36(45.6 \%)$ patients without END $(P=$ 0.001). Patients with APCVS were more likely to have END $(40.0 \%$, vs. $12.2 \%, P=0.001)$ than those without END. Multivariate logistic regression indicated that APCVS (OR $=4.349,95 \%$ C.I. $=1.580-11.970, P=0.004)$ was a significant predictor of END in acute ischemic stroke patients with SIASO, adjusted for previous stroke history and acute infarct volume.

Conclusions: In acute ischemic stroke patients with SIASO, the APCVS might be a useful neuroimaging marker for predicting END, which suggests the importance of evaluation of perfusion status.

Keywords: Acute ischemic stroke, Asymmetrical prominent cortical vein sign, Intracranial arterial stenosis or occlusion, Susceptibility-weighted imaging, Hypoperfusion, Early neurological deterioration

\footnotetext{
* Correspondence: cykun78@163.com

'Department of Neurology, Affiliated Dongguan People's Hospital, Southern Medical University, Wandao Road South No.3, Wanjiang District, Dongguan, Guangdong Province, China

Full list of author information is available at the end of the article
}

(C) The Author(s). 2020 Open Access This article is licensed under a Creative Commons Attribution 4.0 International License, which permits use, sharing, adaptation, distribution and reproduction in any medium or format, as long as you give appropriate credit to the original author(s) and the source, provide a link to the Creative Commons licence, and indicate if changes were made. The images or other third party material in this article are included in the article's Creative Commons licence, unless indicated otherwise in a credit line to the material. If material is not included in the article's Creative Commons licence and your intended use is not permitted by statutory regulation or exceeds the permitted use, you will need to obtain permission directly from the copyright holder. To view a copy of this licence, visit http://creativecommons.org/licenses/by/4.0/. The Creative Commons Public Domain Dedication waiver (http://creativecommons.org/publicdomain/zero/1.0/) applies to the data made available in this article, unless otherwise stated in a credit line to the data. 


\section{Background}

Stroke is the leading cause of death in China $[1,2]$. Intracranial atherosclerosis is a frequent etiology of acute ischemic stroke (AIS) in the Chinese population, with a prevalence of $46.6 \%$ [3]. Patients with AIS caused by intracranial atherosclerosis stenosis or occlusion have a higher risk of early neurological deterioration (END), and the possible underlying mechanisms include hemodynamics, hypoperfusion, or thrombus extension [4-7].

Perfusion status in AIS patients is often evaluated using computed tomography (CT) perfusion or magnetic resonance perfusion, which require additional contrast. In recent years, susceptibility-weighted imaging (SWI) has been applied for the detection of hypoperfusion in AIS patients $[8$, 9]. This high-resolution three-dimensional echo magnetic resonance imaging (MRI) technique is highly sensitive to paramagnetic material such as deoxyhemoglobin and hemosiderin. The asymmetrically prominent cortical vein sign (APCVS) is typically identified as asymmetrical dilatedvessel-like signal loss seen in the cortex on SWI. It is thought to represent either a penumbra or poor collateralization of the arterial supply $[10,11]$. The APCVS is a potential neuroimaging marker for the evaluation of hemodynamics in patients with moyamoya disease [12] and for predicting arterial occlusion in AIS patients [13]. According to Sharma et al. [14], internal cerebral vein asymmetry on follow-up CT angiography is an early predictor of poor functional outcome after intravenous thrombolysis. Another study suggested that the APCVS predicts the clinical course and outcome of anterior circulation ischemic stroke patients in the acute phase and the 90-day prognosis. A positive relationship was also observed between an APCVS and END [15]. However, in most cases, the APCVS occurs in patients with severe intracranial arterial stenosis or occlusion (SIASO), which indicates that SIASO should be considered in the analysis of AIS patients. It is unknown whether an APCVS increases the risk of END in AIS patients with SIASO. Therefore, the purpose of this study was to investigate the relationship between the APCVS and END in AIS patients with SIASO.

\section{Methods}

\section{Participants and setting}

AIS patients admitted to Dongguan People's Hospital between July 1, 2016 and December 31, 2018 were screened retrospectively. The inclusion criteria were (1) age $\geq 18$ years; (2) diagnosis of AIS of the anterior circulation and hospitalized within $24 \mathrm{~h}$ after onset; (3) symptomatic SIASO confirmed by magnetic resonance angiography (MRA). Symptomatic SIASO refers to ipsilateral artery stenosis or occlusion and was defined as more than $70 \%$ diameter loss in any segment of the internal carotid artery (ICA)/middle cerebral artery (MCA) ipsilateral to the infarction. Intracranial stenosis was assessed using the Warfarin Aspirin Symptomatic Intracranial Disease (WASID) Criteria [16]; (4) pre-stroke modified Rankin scale $\leq 1$; and (5) an SWI examination was performed. The exclusion criteria included (1) patients without MRI within $24 \mathrm{~h}$ after admission or those who had poor-quality MRI information; (2) acute infarction involving the posterior circulation or bilateral hemisphere confirmed by diffusion-weighted imaging (DWI); (3) contralateral ICA or MCA stenosis (more than 50\% diameter loss) or occlusion; (4) patients who were discharged early (within 3 days after admission); and (5) incomplete clinical data (e.g., lack of continuous neurological assessment). This study protocol was approved by the Ethics Committee of Dongguan People's Hospital. Consent from the patients was waived because of the retrospective design of the study, which fulfilled the criteria for minimal risk to the patients.

\section{Collection of clinical data}

Demographic characteristics (age and sex) and clinical characteristics, including vascular risk factors (e.g., hypertension, diabetes mellitus, hyperlipidemia, ischemic heart disease, frequent consumption of alcohol, and smoking history), previous stroke history, and treatment information, were collected from patients' medical records. Neurological deficits caused by AIS were assessed using the National Institute of Health Stroke Scale (NIHSS) score recorded from the medical records. All patients received guideline-based treatments after admission.

\section{Definition of END}

In our stroke unit, patients with AIS received a daily NIHSS assessment until $72 \mathrm{~h}$ after admission. The definition of END in our study refers to neurological deterioration with an increase in the NIHSS score $\geq 2$ points in the first $72 \mathrm{~h}$ after admission $[17,18]$.

\section{MRI analysis}

Brain MRI, including T1-weighted imaging, T2-weighted imaging, fluid-attenuated inversion recovery (FLAIR), DWI, SWI, and three-dimensional time-of-flight magnetic resonance angiography (3D-TOF-MRA) was performed for each patient using a $3.0 \mathrm{~T}$ system (Skyra, Siemens Medical, Germany) within $24 \mathrm{~h}$ after admission. Axial spin-echo T1 (TR/TE/excitation $=1500 / 11 / 1$, FOV $=220 \mathrm{~mm}$, slice thickness $/ \mathrm{gap}=4 \mathrm{~mm} / 1.2 \mathrm{~mm}$, matrix $=320 \times 320$, time of acquisition $=1 \mathrm{~min} 26 \mathrm{~s}$ ) and turbo spin-echo T2 (TR/TE/ excitation $=4720 / 96 / 2$, turbo factor $15, \mathrm{FOV}=220 \mathrm{~mm}$, slice thickness $/$ gap $=4$ $\mathrm{mm} / 1.2 \mathrm{~mm}$, matrix of $512 \times 512$, time of acquisition $=1$ min $50 \mathrm{~s}$ ) images were also acquired. Coronal position FLAIR (TR $/$ TE $/$ excitation $=9000 / 84 / 1, \quad F O V=230 \mathrm{~mm}$, slice thickness/gap $=5 \mathrm{~mm} / 1.5 \mathrm{~mm}$, matrix $320 \times 320$, time of acquisition $=1 \mathrm{~min} 50 \mathrm{~s}$ ) and DWI spin-echo 
planar imaging (EPI) (TR/TE/excitation $=4640 / 67 / 1$, matrix $=192 \times 192$, FOV $=230 \mathrm{~mm}$, slice thickness $/$ gap $=$ $4 \mathrm{~mm} / 1.2 \mathrm{~mm}$, EPI factor $=91$, acquisition time $=1 \mathrm{~min}$ $44 \mathrm{~s})$ sequences with three orthogonally applied gradients were used, with b values of 0 and 1000. SWI (TR/ $\mathrm{TE} /$ excitation $=27 / 20 / 1$, FOV $=220 \mathrm{~mm}$, slice thickness $/$ gap $=3 \mathrm{~mm} / 0.6 \mathrm{~mm}$, matrix $256 \times 256$, time of acquisition $=2 \mathrm{~min} 28 \mathrm{~s}$ ) and 3D-TOF-MRA (TR/TE/excitation $=21 / 3.42 / 1, \quad F O V=200 \mathrm{~mm}$, slice thickness $/$ gap $=$ $0.7 \mathrm{~mm} /-0.14 \mathrm{~mm}$, matrix $384 \times 384$, time of acquisition $=3 \min 36 \mathrm{~s}$ ) were also conducted.

An experienced neuroradiologist and a trained neurologist (WF and YKC), who were blinded to the patients' clinical information, independently assessed the MRI variables as well as the APCVS as follows:

1. Infarct. The location, number, and volume of acute infarcts were examined on DWI and the total volume was calculated by multiplying the total area by the sum of the slice thickness and the gap.

2. White matter lesions. The severity of white matter lesions was graded using the four-point scale of Fazekas et al. [19] Deep white matter hyperintensities and periventricular hyperintensities were scored.

3. Intracranial arterial stenosis or occlusion. This was assessed using the WASID Criteria [16].

Symptomatic severe intracranial arterial stenosis was defined as a stenosis $\geq 70 \%$ that affected the ICA or the M1 segment of the MCA ipsilateral to the infarction. Intracranial large artery occlusion was defined as signal loss of distal blood flow.

4. Hemorrhagic transformation. This was confirmed on the basis of signal changes on T1WI, T2WI, and SWI.

5. APCVS. This was the result of an internal comparison of vein signals between the two hemispheres. The APCVS was defined as more and/ or a larger size of cortical veins with greater signal loss on the side with SIASO than on the contralateral size without SIASO in the minimumintensity projection of SWI (Fig. 1) [10]. Ten cases were randomly selected to test the inter-rater and intra-rater agreement, and the results indicated good agreement (inter-rater kappa 0.82; intra-rater kappa 0.86).

\section{Statistical analysis}

Statistical analyses were conducted using SPSS for Windows (v.19.0, SPSS Inc., Chicago, IL, USA). All patients were divided into two groups, the END (+) and END (-) groups. Differences between the groups with and without END were analyzed using Student's $t$-test (for continuous variables), or the chi-squared test or Mann-
Whitney $U$ test (for categorical variables). Variables with $P<0.05$ in the univariate analysis were included as independent variables in the multivariate logistic regression analysis to predict END. Statistical significance was set at $P<0.05$ (two-sided).

\section{Results}

During the study period, 1156 patients with AIS were admitted. Among them, 166 patients had symptomatic SIASO on the side ipsilateral to the acute infarction. We excluded 35 patients receiving intravenous thrombolysis (median NIHSS score: 7.5), 6 patients undergoing thrombectomy (median NIHSS score: 15.5), 10 patients without SWI, and 6 patients without complete clinical data (lack of NIHSS score within $72 \mathrm{~h}$ after stroke). Thus, a total of 109 patients ( 80 men and 29 women) were eventually included in this study, with a mean age of 64.2 (standard deviation, 11.8) years. Compared with the 57 excluded patients with SIASO, the included patients did not differ in age, sex, and NIHSS score at admission $(P>0.05)$. Of these patients, $30 \quad(27.5 \%)$ developed END during the initial $72 \mathrm{~h}$ after admission despite standard treatment. Sixty (55\%) patients presented with an APCVS on SWI.

\section{Relationship between APCVS and END}

The APCVS was present in $24(80 \%)$ patients in the END $(+)$ group, whereas it was found in $36(45.6 \%)$ patients in the END $(-)$ group $(P=0.001)$. However, patients with an APCVS were more likely to have END than those without an APCVS $(40.0 \%$ vs. $12.2 \%, P=$ $0.001)$. Additionally, compared with the END (-) group, the END (+) group had a larger infarct volume on DWI and was more likely to have a previous stroke history (Table 1). After adjusting for infarct volume on DWI and previous stroke history, the multivariate logistic analysis indicated that the APCVS remained a significant predictor of END [OR 4.349, 95\% CI $=1.580-11.970$, $P=0.004]$ (Table 2).

\section{Discussion}

In this study, we found that in AIS patients with SIASO, presence of the APCVS predicted a higher risk of END, which suggests that evaluation of perfusion status with SWI is applicable and helpful for prediction of END. Because of its lack of requirement for a contrast agent and high visibility, the APCVS might be a useful functional imaging marker for END in clinical practice.

END is an important problem in AIS because it is correlated with longer hospitalization and poor prognosis [20-22]. Although several serum biomarkers [23-26], vascular factors [27-31] and neuroimaging parameters $[32,33]$ have been reported to be potential predictors of END, persistent large artery occlusion has been 

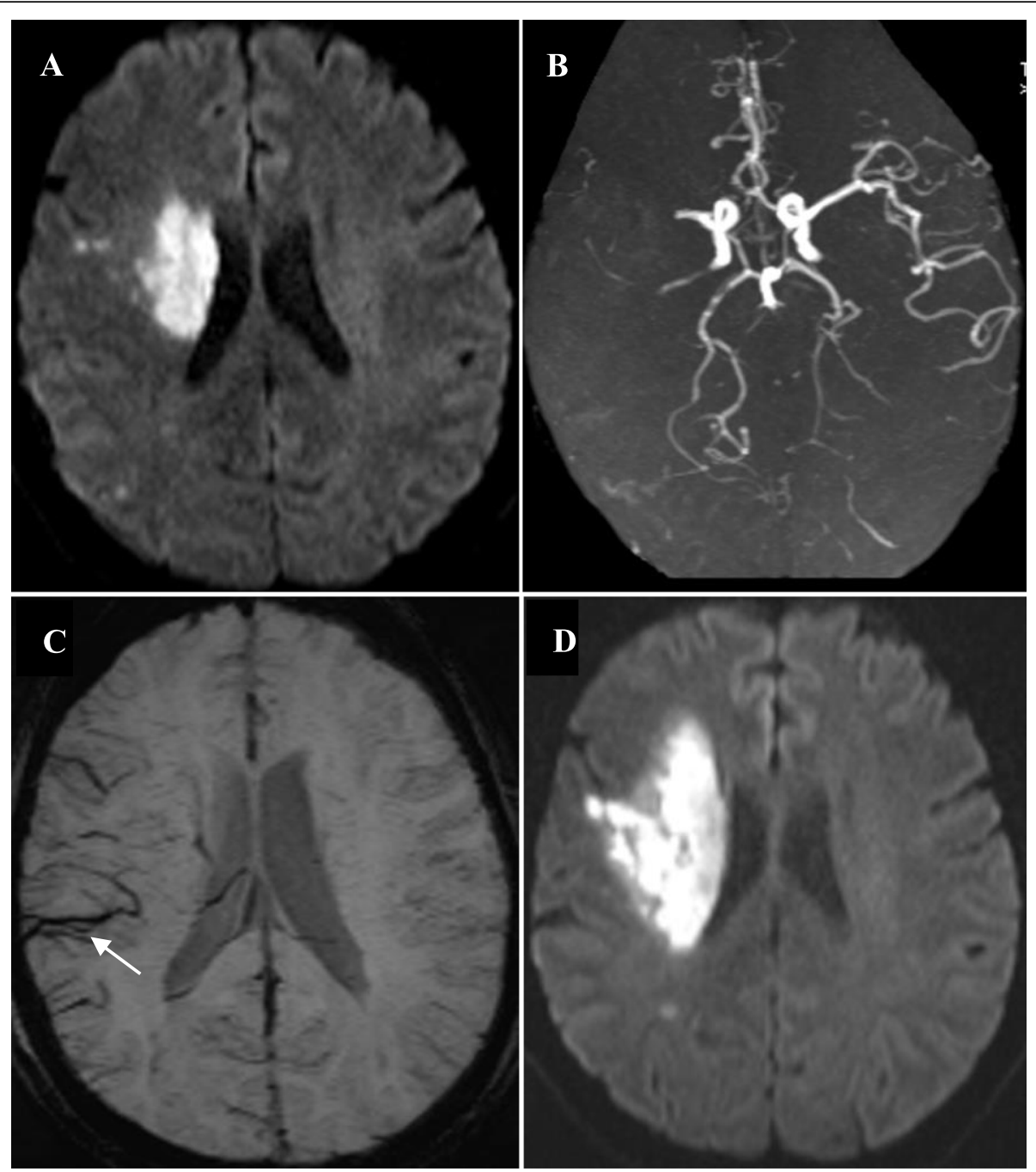

Fig. 1 a Diffusion weighted imaging (DWI) in the first $24 \mathrm{~h}$ after admission: acute infarct in the right basal ganglia in one acute ischemic stroke patient with a National Institutes of Health Stroke Scale (NIHSS) score of 6 at admission. b Magnetic resonance angiography: occlusion in the right middle cerebral artery (MCA). c Susceptibility weighted imaging: asymmetrically prominent cortical vein sign in the right MCA territory (arrow). d Repeat DWI after early neurological deterioration (END), (Day 3 and the NIHSS score increased to 14): enlargement of the infarction

recognized as a major independent risk factor for END. However, many patients with severe large artery stenosis or occlusion have neither a large infarction nor END because of good collateral circulation, and they may not have significant hypoperfusion. In patients with hypoperfusion, the cortical vein presents with a magnetic susceptibility effect that is increased on SWI, which results from the increased deoxyhemoglobin concentration. Several studies have shown that the APCVS is present in AIS patients with unilateral artery stenosis or occlusion $[10,13,34,35]$, and a systematic review reported that the cumulative prevalence of APCVS on SWI in these studies was $81 \%$ (range 34-100\%) [36]. The presence and range of the APCVS in patients with intracranial arterial occlusion has been shown to coincide with hypoperfusion confirmed by magnetic resonance perfusion-weighted imaging [37].

In practice, clinicians are highly concerned about END. However, few studies have examined the relationship between the APCVS and END. Sun et al. [15] found that in AIS patients with MCA territory, the APCVS might be considered a neuroimaging predictor for END. In their study, the APCVS was present in 39 patients, and $37(94.9 \%)$ of these patients had ipsilateral ICA/ MCA stenosis or occlusion. However, the authors did not exclude the effects of ipsilateral ICA/MCA stenosis or occlusion on END because persistent occlusion of the large arteries has been recognized as a major independent risk factor for END. Thus, ipsilateral ICA/MCA stenosis or occlusion should be considered in the 
Table 1 Clinical characteristics of the sample and comparisons of variables between patients with and without END

\begin{tabular}{|c|c|c|c|c|}
\hline & $\begin{array}{l}\text { The whole sample } \\
N=109\end{array}$ & $\begin{array}{l}\text { END (+) } \\
N=30\end{array}$ & $\begin{array}{l}\text { END (-) } \\
N=79\end{array}$ & $P$ \\
\hline \multicolumn{5}{|l|}{ Clinical variables } \\
\hline Age $^{*}$ & $64.25 \pm 11.78$ & $66.4 \pm 11.43$ & $64.43 \pm 11.88$ & 0.242 \\
\hline Male $n(\%)+$ & $80(73.4 \%)$ & $22(73.3 \%)$ & $58(73.4 \%)$ & 0.993 \\
\hline Hypertension n(\%)+ & $81(73.2 \%)$ & $22(73.3 \%)$ & $59(74.7 \%)$ & 0.885 \\
\hline Diabetes melitus n(\%)+ & $40(36.7 \%)$ & $14(46.7 \%)$ & $26(32.9 \%)$ & 0.183 \\
\hline Hyperlipidemia, n(\%)+ & $40(36.7 \%)$ & $9(30 \%)$ & $31(39.2 \%)$ & 0.505 \\
\hline Ischemic heart disease, $n(\%)^{n}$ & $7(6.4 \%)$ & $1(3.3 \%)$ & $6(7.6 \%)$ & 0.671 \\
\hline Atrial fibrillation $n(\%)^{q}$ & $12(11.0 \%)$ & $2(6.7 \%)$ & $10(12.7 \%)$ & 0.505 \\
\hline Previous stroke $\mathrm{n}(\%)^{n}$ & $27(24.8 \%)$ & $3(10 \%)$ & $24(30.4 \%)$ & 0.028 \\
\hline Frequent consumption of alcohol n(\%) & $11(10.1 \%)$ & $2(6.7 \%)$ & $9(11.4 \%)$ & 0.37 \\
\hline Smoking $n(\%)+$ & $53(48.6 \%)$ & $12(40 \%)$ & $41(51.9 \%)$ & 0.267 \\
\hline NIHSS score at admission ${ }^{\S}$ & $4(2-8)$ & $4(2-7.25)$ & $5(2-8)$ & 0.379 \\
\hline SBP at admission* & $154.46 \pm 21.88$ & $158.6 \pm 19.9$ & $152.9 \pm 22.5$ & 0.221 \\
\hline DBP at admission* & $89.7 \pm 13.7$ & $90.0 \pm 15.0$ & $89.6 \pm 12.3$ & 0.875 \\
\hline Prestroke usage of antiplatelet agents, n(\%) & $17(15.6 \%)$ & $2(6.7 \%)$ & $15(19.0 \%)$ & 0.146 \\
\hline Prestroke usage of statins, n (\%) & $14(12.8 \%)$ & $2(6.7 \%)$ & $12(15.2 \%)$ & 0.342 \\
\hline Prestroke usage of antihypertensive agents, n (\%) & $31(28.4 \%)$ & $9(30 \%)$ & $22(27.8 \%)$ & 0.824 \\
\hline Prestroke usage of glucose lowering agents, n (\%) & $14(13.4 \%)$ & $6(20 \%)$ & $8(10.1 \%)$ & 0.169 \\
\hline Prestroke usage of anticoagulants, n (\%) & $3(2.7 \%)$ & 0 & $3(3.7 \%)$ & 0.560 \\
\hline Dual-antiplatelet agents after admission, n (\%) & $41(37.6 \%)$ & $13(43.3 \%)$ & $28(35.4 \%)$ & 0.448 \\
\hline \multicolumn{5}{|l|}{ MRI variables } \\
\hline DWl-infarct volume $\left(\mathrm{mm}^{3}\right)^{\S}$ & $10.3(2.3-19.7)$ & $14.2(6.6-21.9)$ & $7.7(1.6-18.2)$ & 0.037 \\
\hline Hemorrhagic transformation $n(\%)^{n}$ & $13(11.9 \%)$ & $3(10 \%)$ & $10(12.7 \%)$ & 1.000 \\
\hline $\mathrm{WMHs}^{\S}$ & $2(0-2.0)$ & $2(0-2.25)$ & $2(0-2.0)$ & 0.847 \\
\hline APCVS n(\%) & $60(55 \%)$ & $24(80 \%)$ & $36(45.6 \%)$ & 0.001 \\
\hline
\end{tabular}

END Early Neurological Deterioration, NIHSS National Institutes of Health Stroke Scale, SBP Systolic Blood Pressure, DBP Diastolic Blood Pressure, DWI Diffusion Weighted Imaging, WMHs White Matter Hyperintensities, APCVS Asymmetrically Prominent Cortical Vein Sign

*represents mean \pm SD, t-test; +represents n\%, chi-square test; १ represents n\%, Fisher exact test; $\S$ represents median (25Q-75Q), Mann-Whiteney U test

analysis of these patients. Considering this confounding factor, our research was focused on SIASO patients, and AIS patients with contralateral ICA or MCA stenosis (more than $50 \%$ diameter loss) or occlusion were excluded because the APCVS results were from a comparison within an individual [38]. Another study reported [39] that a peripheral APCVS was positively correlated with the degree of MCA stenosis, and fewer peripheral APCVSs may suggest a favorable outcome of unilateral MCA infarction at the 3-month follow-up. However,

Table 2 Multivariate logistic regression of risk factors for poor outcome

\begin{tabular}{llll}
\hline Variables & $\beta$ & OR(95\%C.I.) & $P$ \\
\hline DWl-infarct volume $\left(\mathrm{mm}^{3}\right)$ & -0.005 & $0.995(0.997-1.014)$ & 0.615 \\
Previous stroke & -1.196 & $0.302(0.080-1.136)$ & 0.077 \\
APCVS & 1.470 & $4.349(1.580-11.970)$ & 0.004 \\
\hline
\end{tabular}

APCVS Asymmetrically Prominent Cortical Vein Sign they did not elaborate on the relationship between the APCVS and AIS patients in the acute phase.

Our study found that patients with an APCVS had a higher risk for END $(\mathrm{OR}=4.3)$ on the basis of symptomatic SIASO, even after adjusting for possible confounders, which suggests that the APCVS is a reliable imaging marker for END.

There are several limitations to this study. First, the retrospective design could not confirm a causal relationship between APCVS and END. We did not follow up on patients after their admission and did not investigate 3-month or long-term outcomes. Future studies will therefore explore whether the APCVS can predict the recurrence of ischemic stroke or intracranial hemorrhage. Second, the APCVS was not evaluated with a satisfactory quantitative measurement. We did not measure the medullary vein sign, another SWI marker of hypoperfusion, in this study. In the future, autonomic computed assessment tools are therefore warranted to 
achieve a more accurate and reliable result. Third, we were unable to classify the exact reason for END and for patients with fluctuating NIHSS scores in this retrospective study. Fourth, it should be noted that the findings in this study are limited to the target subjects set by the inclusion and exclusion criteria and are not applicable to patients with bilateral SIASO.

\section{Conclusion}

We demonstrated that the APCVS is a strong neuroimaging marker for END in AIS patients with ipsilateral SIASO, which suggests the importance of evaluating perfusion status. SWI should be a routine MRI sequence in patients with AIS because of its capacity to safely and conveniently assess perfusion status.

\section{Abbreviations}

SWI: Susceptibility weighted imaging; END: Early neurological deterioration; SIASO: Severe intracranial arterial stenosis or occlusion; NIHSS: National Institutes of Health Stroke Scale; AIS: Acute ischemic stroke; DWI: Diffusion weighted imaging; ICA: Internal carotid artery; MCA: Middle cerebral artery; APCVS: Asymmetrical prominent cortical vein sign; WASID: Warfarin Aspirin Symptomatic Intracranial Disease

\section{Acknowledgements}

We thank Lisa Kreiner, PhD, from Liwen Bianji, Edanz Editing China (www. liwenbianji.cn/ac), for editing the English text of a draft of this manuscript.

\section{Authors' contributions}

YKC and WMX designed the work. WL, GPL, YLL and JFQ collected the clinical data, discussed the results. XWF trained the MRI assessment. FW and YKC assessed the MRI variables. WL and YKC wrote the manuscript. All authors read and approved the final manuscript; contributed toward data analysis, drafted and revised the paper, and agreed to be accountable for all aspects of the work.

\section{Funding}

This study was funded by the Science and Technology Planning Project of Guangdong Province of China (2017A020215002). The funding body had no role in the design of the study, collection, analysis, and interpretation of the data, and in writing of the manuscript.

\section{Availability of data and materials}

The datasets generated and/or analyzed during the current study are not publicly available because they are personal data, but they are available from the corresponding author on reasonable request.

\section{Ethics approval and consent to participate}

This study protocol was approved by the Ethics Committee of Dongguan People's Hospital. As this was a retrospective study, consent to participate was waived.

\section{Consent for publication}

As this was a retrospective study, consent for publication was waived.

\section{Competing interests}

The authors declare that they have no competing interests.

\section{Author details}

'Department of Neurology, Affiliated Dongguan People's Hospital, Southern Medical University, Wandao Road South No.3, Wanjiang District, Dongguan, Guangdong Province, China. ${ }^{2}$ Department of Radiology, Affiliated Dongguan People's Hospital, Southern Medical University, Dongguan, Guangdong Province, China.
Received: 24 April 2020 Accepted: 24 August 2020

Published online: 02 September 2020

\section{References}

1. Liu L, Wang D, Wong KSL, Wang YJ. Stroke and stroke care in China: huge burden, significant workload, and a national priority. Stroke. 2011;42(12): 3651-4

2. Wu S, Wu B, Liu M, Chen ZM, Wang WZ, Anderson CS, Sandercock P, Wang YJ, Huang YM, Cui LY, et al. Stroke in China: advances and challenges in epidemiology, prevention, and management. Lancet Neurol. 2019;18(4):394405.

3. Wang YJ, Zhao XQ, Liu LP, Soo YOY, Pu YH, Pan YS, Wang YL, Zou XY, Leung TWH, Cai YF, et al. Prevalence and outcomes of symptomatic intracranial large artery stenoses and occlusions in China: the Chinese Intracranial Atherosclerosis (CICAS) study. Stroke. 2014;45(3):663-9.

4. Alawneh JA, Moustafa RR, Baron JC. Hemodynamic factors and perfusion abnormalities in early neurological deterioration. Stroke. 2009:40(6):e443-50.

5. Seners $P$, Hurford R, Tisserand M, Turc G, Legrand L, Naggara $O$, Mas JL, Oppenheim C, Baron JC. Is unexplained early neurological deterioration after intravenous thrombolysis associated with thrombus extension? Stroke. 2017:48(2):348-52.

6. Ois A, Rordriguez JEM, Munteis E, Gomis M, Campello AR, Conde JJ, Godia EC, Roquer J. Steno-occlusive arterial disease and early neurological deterioration in acute ischemic stroke. Cerebrovasc Dis. 2008;25(1-2):151-6.

7. Derdeyn CP. Mechanisms of ischemic stroke secondary to large artery atherosclerotic disease. Neuroimaging Clin N Am. 2007;17(3):303-11 vii-viii.

8. Park MG, Yang TI, Oh SJ, Baik SK, Kang YH, Park KP. Multiple hypointense vessels on susceptibility-weighted imaging in acute ischemic stroke: surrogate marker of oxygen extraction fraction in penumbra? Cerebrovasc Dis. 2014;38(4):254-61.

9. Hsu CCT, Kwan GNC, Hapugoda S, Craigie M, Watkins TW, Haacke EM. Susceptibility weighted imaging in acute cerebral ischemia: review of emerging technical concepts and clinical applications. Neuroradiol J. 2017; 30(2):109-19.

10. Baik SK, Choi WJ, Oh SJ, Park KP, Park MG, Yang TI, Jeong HW. Change in cortical vessel signs on susceptibility-weighted images after full recanalization in hyperacute ischemic stroke. Cerebrovasc Dis. 2012;34(3): 206-12.

11. Fujioka M, Okuchi K, Iwamura A, Takka T, Siesjo BK. A mismatch between the abnormalities in diffusion- and susceptibility-weighted magnetic resonance imaging may represent an acute ischemic penumbra with misery perfusion. J Stroke Cerebrovasc Dis. 2013;22(8):1428-31.

12. Wei QQ, Shan TK, Qiu QQ, Zhang JG, Xia CC, Liu Y, Yuou C. Asymmetric cortical vessel sign indicates hemodynamic deficits in adult patients with moyamoya disease. World Neurosurg. 2019;127:e137-41.

13. Payabvash S, Benson JC, Taleb S, Rykken JB, Hoffman B, Oswood MC, McKinney AM. Prominent cortical and medullary veins on susceptibilityweighted images of acute ischaemic stroke. Br J Radiol. 2016;89(1068): 20160714.

14. Sharma VK, Yeo LLL, Teoh HL, Liang Shen L, Chan BPL, Seet RC, Ahmad A, Chong VF, Paliwal PR. Internal cerebral vein asymmetry on follow-up brain computed tomography after intravenous thrombolysis in acute anterior circulation ischemic stroke is associated with poor outcome. J Stroke Cerebrovasc Dis. 2014:23(1):e39-45.

15. Sun W, Liu W, Zhang Z, Xiao L, Duan Z, Liu D, Xiong Y, Zhu W, Lu G, Liu X. Asymmetrical cortical vessel sign on susceptibility-weighted imaging: a novel imaging marker for early neurological deterioration and unfavorable prognosis. Eur J Neurol. 2014;21(11):1411-8.

16. The Warfarin-Aspirin Symptomatic Intracranial Disease (WASID) Study Group Prognosis of patients with symptomatic vertebral or basilar artery stenosis. Stroke. 1998;29(7):1389-92.

17. Siegler JE, Schild SM. Early Neurological seterioration (END) after stroke: the END depends on the definition. Int J Stroke. 2011;6(3):211-2.

18. Chen ZL, Li W, Sun W, Xiao LL, Dai QL, Cao YZ, Han YF, Zhu WS, Xu GL, Liu XF. Correlation study between small vessel disease and early neurological deterioration in patients with mild/moderate acute ischemic stroke. Int J Neurosci. 2017:127(7):579-85.

19. Fazekas F, Chawluk JB, Alavi A, Hurtig HI, Zimmerman RA. MR signal abnormalities at $1.5 \mathrm{~T}$ in Alzheimer's dementia and normal aging. AJR Am J Roentgenol. 1987;149(2):351-6. 
20. Toni D, Fiorelli M, Gentile M, Bastianello S, Sacchetti ML, Argentina C, Pozzilli C, Fieschi C. Progressing neurological deficit secondary to acute ischemic stroke. A study on predictability, pathogenesis, and prognosis. Arch Neurol. 1995;52(7):670-5.

21. Steinke W, Ley SC. Lacunar stroke is the major cause of progressive motor deficits. Stroke. 2002;33(6):1510-6.

22. Bene AD, Palunma V, Lamassa M, Saia V, Piccardi B, Inzitari D. Progressive lacunar stroke: review of mechanisms, prognostic features, and putative treatments. Int J Stroke. 2012;7(4):321-9.

23. Seo WK, Palumbo V, Lamassa M, Saia V, Piccardi B, Inzitari D. C-reactive protein is a predictor of early neurologic deterioration in acute ischemic stroke. J Stroke Cerebrovasc Dis. 2012;21(3):181-6.

24. Suda S, Katsumata T, Okubo S, Kanamaru T, Suzuki K, Watanabe Y, Katsura KI, Katayama Y. Low serum n-3 polyunsaturated fatty acid/n-6 polyunsaturated fatty acid ratio predicts neurological deterioration in Japanese patients with acute ischemic stroke. Cerebrovasc Dis. 2013;36(5-6): 388-93.

25. Kwon HM, Lee YS, Bae HJ, Kang DW. Homocysteine as a predictor of early neurological deterioration in acute ischemic stroke. Stroke. 2014:45(3):871-3.

26. Wang Y, Hu SY, Ren LJ, Lei ZH, Lan T, Cai JJ, Li C. Lp-PLA2 as a risk factor of early neurological deterioration in acute ischemic stroke with TOAST type of large arterial atherosclerosis. Neurol Res. 2019:41(1):1-8

27. Cuadrado-Godia E, Jimena S, Ois A, Rodriguez-Campllo A, Giralt-Steinhauer E, Soriano-Tarraga C, Jimenez-Conde J, Martinez-Rodriquez JE, Capellades J, Roquer J. Factors associated with early outcome in patients with largevessel carotid strokes. J Neurol Neurosurg Psychiatry. 2013;84(3):305-9.

28. Nacu A, Bringeland GH, Khanevski A, Thomassen L, Waje-Andreassen U, Naess $\mathrm{H}$. Early neurological worsening in acute ischaemic stroke patients. Acta Neurol Scand. 2016;133(1):25-9.

29. Ois A, Cuadrado-Godia E, Rodriguez-Campello A, Jimenez-Conde J, Roquer J. High risk of early neurological recurrence in symptomatic carotid stenosis. Stroke. 2009;40(8):2727-31.

30. Burke MJ, Vergouwen MDI, Fang JM, Swartz RH, Kapal MK, Silver FL, Casaubon LK. Investigators of the registry of the Canadian stroke network. Short-term outcomes after symptomatic internal carotid artery occlusion. Stroke. 2011;42(9):2419-24.

31. Tei H, Uchiyama S, Ohara K, Kobayashi M, Uchiyama Y, Fukuzawa M. Deteriorating ischemic stroke in 4 clinical categories classified by the Oxfordshire community stroke project. Stroke. 2000;31(9):2049-54

32. Jeong HG, Kim BJ, Yang MH, Han MK, Bae HJ. Neuroimaging markers for early neurologic deterioration in single small subcortical infarction. Stroke. 2015:46(3):687-91.

33. Arenillas JF, Rovira A, Molina CA, Grive E, Montaner J, Alvarez-Sabin J. Prediction of early neurological deterioration using diffusion- and perfusionweighted imaging in hyperacute middle cerebral artery ischemic stroke. Stroke. 2002;33(9):2197-203.

34. Huang PY, Chen CH, Lin WC, Lin RT, Khor GT, Liu CK. Clinical applications of susceptibility weighted imaging in patients with major stroke. J Neurol. 2012;259(7):1426-32.

35. Kesavadas C, Thomas B, Pendharakar H, Sylaja PN. Susceptibility weighted imaging: does it give information similar to perfusion weighted imaging in acute stroke? J Neurol. 2011;258(5):932-4.

36. Jensen-Kondering $\mathrm{U}$, Bohm B. Asymmetrically hypointense veins on $\mathrm{T2}^{*} \mathrm{~W}$ imaging and susceptibility-weighted imaging in ischemic stroke. World J Radiol. 2013;5(4):156-65.

37. Mundiyanapurath $S$, Ringleb PA, Diatschuk S, Burth S, Mohlenbruch M, Floca FO, Wick W, Bendszus M, Radbruch A. Cortical vessel sign on susceptibility weighted imaging reveals clinically relevant hypoperfusion in internal carotid artery stenosis. Eur J Radiol. 2016;85(3):534-9.

38. Xia S, Utriainen D, Tang J, Kou ZF, Zheng G, Wang XS, Shen W, Haacke EM, Lu GM. Decreased oxygen saturation in asymmetrically prominent cortical veins in patients with cerebral ischemic stroke. Magn Reson Imaging. 2014; 32(10):1272-6

39. Yuan T, Ren GL, Quan GM, Gao D. Fewer peripheral asymmetrical cortical veins is a predictor of favorable outcome in MCA infarctions with SWI-DWI mismatch. J Magn Reson Imaging. 2018;48(4):964-70.

\section{Publisher's Note}

Springer Nature remains neutral with regard to jurisdictional claims in published maps and institutional affiliations.

\section{Ready to submit your research? Choose BMC and benefit from:}

- fast, convenient online submission

- thorough peer review by experienced researchers in your field

- rapid publication on acceptance

- support for research data, including large and complex data types

- gold Open Access which fosters wider collaboration and increased citations

- maximum visibility for your research: over $100 \mathrm{M}$ website views per year

At $\mathrm{BMC}$, research is always in progress.

Learn more biomedcentral.com/submissions 